VOL. 60 (1999) [207-220]

\title{
FINITE NORMAL EDGE-TRANSITIVE CAYLEY GRAPHS
}

\author{
Cheryl E. Praeger
}

\author{
To Bernhard Neumann, my mentor and friend, \\ with love and admiration, on the occasion of his 90th birthday
}

\begin{abstract}
An approach to analysing the family of Cayley graphs for a finite group $G$ is given which identifies normal edge-transitive Cayley graphs as a sub-family of central importance. These are the Cayley graphs for $G$ for which a subgroup of automorphisms exists which both normalises $G$ and acts transitively on edges. It is shown that, for a nontrivial group $G$, each normal edge-transitive Cayley graph for $G$ has at least one homomorphic image which is a normal edge-transitive Cayley graph for a characteristically simple quotient group of $G$. Moreover, given a normal edge-transitive Cayley graph $\Gamma_{H}$ for a quotient group $G / H$, necessary and sufficient conditions are obtained for the existence of a normal edge-transitive Cayley graph $\Gamma$ for $G$ which has $\Gamma_{H}$ as a homomorphic image, and a method for obtaining all such graphs $\Gamma$ is given.
\end{abstract}

\section{INTRODUCTION}

For a group $G$, and a subset $S$ of $G$ such that $1_{G} \notin S$, the Cayley graph Cay $(G, S)$ of $G$ relative to $S$ is defined as the graph with vertex set $G$ and edge set $E(S)$ consisting of those ordered pairs $(x, y)$ from $G$ for which $y x^{-1} \in S$. If $S$ is symmetric, that is, if $S^{-1}:=\left\{s^{-1}: s \in S\right\}$ is equal to $S$, then $(x, y)$ is an edge if and only if $(y, x)$ is an edge, and $\operatorname{Cay}(G, S)$ is said to be undirected. The graph $\operatorname{Cay}(G, S)$ is vertex-transitive since it admits $G$, acting by right multiplication, as a subgroup of automorphisms. Thus $G \leqslant \operatorname{Aut}(\operatorname{Cay}(G, S))$ and this action of $G$ is regular on vertices, that is, $G$ is transitive on vertices and only the identity element of $G$ fixes a vertex. A graph $\Gamma$ is (isomorphic to) a Cayley graph for some group if and only if its automorphism group $\operatorname{Aut}(\Gamma)$ has a subgroup which is regular on vertices, (see [2, Lemma 16.3]). For small values of $n$, the vast majority of undirected vertex-transitive graphs with $n$ vertices are Cayley graphs (see $[8$, Table 1]).

Received 27th April, 1999

We are grateful to Cai Heng Li for his many helpful comments on the first draft of this paper.

The development of the theory of normal edge-transitive Cayley graphs was a research project funded by an Australian Research Council small grant.

Copyright Clearance Centre, Inc. Serial-fee code: 0004-9727/99 \$A2.00+0.00. 
A Cayley graph $\Gamma=\operatorname{Cay}(G, S)$ is said to be edge-transitive if Aut $(\Gamma)$ is transitive on edges. Also, if $\Gamma$ is undirected, then an unordered pair of edges $\{(x, y),(y, x)\}$ is called an unordered edge, and $\Gamma$ is said to be edge-transitive as an undirected graph if Aut $(\Gamma)$ is transitive on unordered edges. In this paper we present an approach to studying the family of Cayley graphs for a given finite group $G$, which focuses attention on those graphs $\Gamma$ for which $N_{\text {Aut }(\Gamma)}(G)$ is transitive on edges, and those undirected graphs $\Gamma$ for which $N_{\mathrm{Aut}(\Gamma)}(G)$ is transitive on unordered edges. Such a graph is said to be normal edge-transitive, or normal edge-transitive as an undirected graph, respectively. Not every edge-transitive Cayley graph is normal edge-transitive. This can be seen by considering the complete graphs $K_{n}$ on $n$ vertices.

EXAMPLE 1. The complete graph $K_{n}$ is an undirected Cayley graph for any group of order $n$, and its automorphism group $S_{n}$ acts transitively on edges, and hence also on unordered edges. However $K_{n}$ is normal edge-transitive (and also normal edge-transitive as an undirected graph) if and only if $n$ is a prime power. If $n=p^{a}$ ( $p$ a prime and $a \geqslant 1)$, then taking $G=\mathrm{Z}_{p}^{a}$ we have $K_{n} \cong \operatorname{Cay}(G, G \backslash\{1\})$ and $N_{S_{n}}(G)=\operatorname{AGL}(a, p)$ is transitive on edges (and on undirected edges).

However in most situations, it is difficult to find the full automorphism group of a graph. Although we know that a Cayley $\operatorname{graph} \operatorname{Cay}(G, S)$ is vertex-transitive, simply because of its definition, in general it is difficult to decide whether it is edge-transitive. On the other hand we often have sufficient information about the group $G$ to determine $N=N_{\text {Aut (Cay }(G, S))}(G)$; for $N$ is the semidirect product

$$
N=G \cdot \operatorname{Aut}(G ; S), \text { where } \operatorname{Aut}(G ; S):=\left\{\sigma \in \operatorname{Aut}(G) \mid S^{\sigma}=S\right\}
$$

Thus it is often possible to determine whether $\operatorname{Cay}(G, S)$ is normal edge-transitive.

Independently of our investigation, and as another attempt to study the structure of finite Cayley graphs, Xu [12] defined a Cayley graph $\Gamma=\operatorname{Cay}(G, S)$ to be normal if $G$ is a normal subgroup of the full automorphism group Aut $(\Gamma)$. Xu's concept of normality for a Cayley graph is a very strong condition. For example, $K_{n}$ is normal if and only if $n \leqslant 4$. However any edge-transitive Cayley graph which is normal, in the sense of $\mathrm{Xu}$ 's definition, is automatically normal edge-transitive. This provides several more families of examples of normal edge-transitive Cayley graphs. For example, the normal Cayley graphs on $2 p$ vertices ( $p$ a prime) which are not undirected were completely classified in [4]. Also it was shown in [1] that most small valency Cayley graphs for finite Abelian groups are normal. Each of these families contains some normal edge-transitive Cayley graphs.

In Section 2 we show how to express an arbitrary Cayley graph for $G$ as an edgedisjoint union of normal edge-transitive Cayley graphs for $G$. Moreover if the original graph $\Gamma$ is undirected then we write $\Gamma$ as an edge-disjoint union of undirected Cayley graphs for $G$, all of which are normal edge-transitive as undirected graphs (see The- 
orem 2). We obtain in Proposition 1 necessary and sufficient conditions in terms of $\operatorname{Aut}(G ; S)$ for $\operatorname{Cay}(G, S)$ to be normal edge-transitive. We illustrate this with the family of Cayley graphs for a cyclic group of prime order. (The assertions made in Example 2 can easily be deduced from Chao's classification of symmetric graphs of prime order in [3]. Details are also given in [6, Section 5.2].)

EXAmple 2. Let $G=\mathrm{Z}_{p}$ denote the additive group of integers modulo a prime $p$. Let $x$ be a generator of $\operatorname{Aut}(G)$, which we identify with the cyclic multiplicative group of nonzero integers modulo $p$, and for each $i$ let $\left\langle x^{i}\right\rangle$ denote the subgroup of $\operatorname{Aut}(G)$ generated by $x^{i}$. Note that, as a set, $\left\langle x^{i}\right\rangle \subseteq G \backslash\{0\}$, and that $\left\langle x^{i}\right\rangle$ is symmetric, as a subset of $G$, if and only if it contains $-x^{i}$, that is, if and only if either $p=2$ or $\operatorname{gcd}(p-1, i)$ divides $(p-1) / 2$. For each divisor $a$ of $p-1$, the Cayley graph $\Gamma(p ; a):=\operatorname{Cay}\left(G,\left\langle x^{(p-1) / a}\right\rangle\right)$ has out-valency $a$, and is normal edge-transitive with $N_{\text {Aut }(\Gamma(p ; a)\rangle}(G)=G \cdot\left\langle x^{(p-1) / a}\right\rangle$. Moreover, $\Gamma(p ; a)$ is undirected if and only if either $p=2$ or $a$ is even. Conversely, each normal edge-transitive Cayley graph for $G$ is either an empty graph, or is isomorphic to $\Gamma(p ; a)$ for some divisor $a$ of $p-1$, and a non-empty undirected Cayley graph for $G$, which is normal edge-transitive as an undirected graph, is isomorphic to $\Gamma(p ; a)$ where either $p=2$ or $a$ is even. For an arbitrary Cayley graph $\Gamma=\operatorname{Cay}(G, S)$ for $G$, we have $N_{\mathrm{Aut}(\Gamma)}(G)=G \cdot\left\langle x^{(p-1) / a}\right\rangle$ for some $a$, and $\Gamma$ is the edge-disjoint union of graphs isomorphic to $\Gamma(p ; a)$.

In Section 3, we investigate quotients of Cayley graphs and determine conditions under which the quotient is a Cayley graph for a quotient group, and conditions for the quotient to be normal edge-transitive. We prove in Theorem 3 that a group $G$ induces a group of automorphisms of a quotient of $\operatorname{Cay}(G, S)$ relative to a vertex partition $\mathcal{P}$ if and only if $\mathcal{P}$ is the set of cosets of a subgroup $H$ of $G$, and that the quotient is a Cayley graph for a quotient group of $G$ if and only if $H$ is a normal subgroup. In this case the quotient is $\operatorname{Cay}(G / H, S H / H)$, and is undirected if $\operatorname{Cay}(G, S)$ is undirected. If $G \cdot \operatorname{Aut}(G ; S)$ is transitive on edges (or on unordered edges if $\operatorname{Cay}(G, S)$ is undirected), then $G \cdot \operatorname{Aut}(G ; S)$ acts transitively on edges (or unordered edges) of the quotient if and only if $H$ is $\operatorname{Aut}(G ; S)$-invariant. This will be the case in particular if $H$ is a characteristic subgroup of $G$.

Thus, given a normal edge-transitive Cayley graph $\operatorname{Cay}(G, S)$ for a nontrivial finite group $G$, we obtain a non-empty set $\mathcal{C}(G, S)$ of normal edge-transitive Cayley graphs for certain quotient groups of $G$. We show in Theorem 4 that every graph $\operatorname{Cay}(G / L, S L / L) \in \mathcal{C}(G, S)$ has at least one homomorphic image which is a graph $\operatorname{Cay}(G / H, S H / H) \in \mathcal{C}(G, S)$ with $H$ a maximal Aut $(G ; S)$-invariant subgroup of $G$. For such a 'minimal' element $\operatorname{Cay}(\widehat{G}, \widehat{S})=\operatorname{Cay}(G / H, S H / H)$, the group $\widehat{G}=G / H$ is characteristically simple (which we define just before Theorem 4) and is a minimal normal subgroup of $\widehat{N}:=\widehat{G} \cdot \operatorname{Aut}(\widehat{G}: \widehat{S})$. Unfortunately, this latter property does not imply that $\widehat{N}$ acts quasiprimitively on $\widehat{G}$ since $\widehat{N}$ may have an intransitive normal subgroup 
which centralises $\widehat{G}$. (A permutation group $G$ on $\Omega$ is said to be quasiprimitive on $\Omega$ if every nontrivial normal subgroup of $G$ is transitive on $\Omega$.) However, quasiprimitive permutation groups with regular normal subgroups provide a large class of examples of normal edge-transitive Cayley graphs $\operatorname{Cay}(\widehat{G}, \widehat{S})$ with $\widehat{G}$ characteristically simple. The quasiprimitive permutation groups are well understood (see $[9,10]$ ), and fall naturally into eight disjoint classes, as described in [10, Section 5]. Each quasiprimitive group in four of these classes very naturally gives rise to examples of normal edge-transitive Cayley graphs for a characteristically simple group. These classes are labelled HA, HS, $\mathrm{HC}$, and TW, and groups in these classes have characteristically simple regular normal subgroups; one simply takes any non-trivial orbital graph of a quasiprimitive group in one of these classes. Groups in two of the remaining four classes, namely the classes $\mathrm{SD}$ and $\mathrm{CD}$, visibly contain characteristically simple regular subgroups which are not normal, and consequently all orbital graphs for quasiprimitive groups in these two classes will also be edge-transitive Cayley graphs for characteristically simple groups, but they may or may not be normal edge-transitive.

Thus normal-edge transitive Cayley graphs for finite characteristically simple groups are of particular importance, as is the relationship between the graphs in $\mathcal{C}(G, S)$ and the original graph $\operatorname{Cay}(G, S)$ for general finite groups $G$. The analysis presented in this paper suggests that the following questions concerning the automorphism groups of normal edge-transitive Cayley graphs deserve further attention.

QUeSTion 1. Construction. Given a normal edge-transitive Cayley graph Cay $(\widehat{G}, \widehat{S})$ (possibly undirected), and a group $G$ with normal subgroup $H$ such that $G / H=\widehat{G}$, when is it possible to construct a Cayley graph $\operatorname{Cay}(G, S)$ with $S H / H=\widehat{S}$ such that there is a group $N$ which normalises $G$, leaves $H$ invariant, and is transitive on edges (or unordered edges if $\operatorname{Cay}(G, S)$ is undirected)? If there is such a graph, give a description of the family of all such graphs. When is there such a graph which is a cover of Cay $(\widehat{G}, \widehat{S})$ ?

QUESTION 2. Reconstruction. Given a normal edge-transitive Cayley graph Cay $(G, S)$, under what conditions is it determined by its quotient graphs in $\mathcal{C}(G, S)$ ?

The simplest cases of Questions 1 and 2 have been addressed by Pantazis Houlis in. his MSc thesis [6]. There the case where $G / H$ is cyclic of prime order, and $G$ is Abelian of order a product of two primes has been analysed completely. In Section 4, we give a general answer for Question 1, which may be used to obtain explicit lists of possibilities for individual groups or families of groups, see Theorem 5. As an application, we discuss the strategy used by Houlis to obtain his classification results.

QUESTION 3. Characteristically simple Cayley graphs. Give a useful description of the family of connected normal edge-transitive Cayley graphs for finite characteristically simple groups.

There are many interesting examples of such graphs. For example, in [7] it was 
proved that all connected Cayley graphs Cay $(\operatorname{PSL}(2, q), S)$ with $|S|=2$ are normal; many of these are edge-transitive and hence are normal edge-transitive. (The connected component containing $1_{G}$ of $\operatorname{Cay}(G, S)$ is the collection of elements of $G$ expressible as a product $s_{1} s_{2} \ldots s_{r}$ of elements $s_{i} \in S$, and hence for a finite group $G, \operatorname{Cay}(G, S)$ is connected if and only if $S$ is a generating set for $G$.) It would be interesting, for example, to know whether every connected Cayley graph of valency 2 for a given nonabelian simple group is normal.

QUESTION 4. Full automorphism group. For a normal edge-transitive Cayley graph $\Gamma=\operatorname{Cay}(G, S)$, how much information about $\operatorname{Aut}(\Gamma)$ is contained in its subgroup $\operatorname{Aut}(G ; S)$ ? In particular when can we guarantee that $G \cdot \operatorname{Aut}(G ; S)$ is not much smaller than $\operatorname{Aut}(\Gamma)$ ?

QUESTION 5. Edge-transitivity. What can be said about the structure of Cayley graphs $\Gamma$ which are edge-transitive but not normal edge-transitive?

These two questions seek information about the possible non-normality of edgetransitive Cayley graphs. The complete graphs of prime power order discussed in Example 1 provide examples of normal edge-transitive Cayley graphs for which the full automorphism group is much larger than $G \cdot \operatorname{Aut}(G ; S)$. We are seeking general conditions which might guarantee that $G \cdot \operatorname{Aut}(G ; S)$ is close to $\operatorname{Aut}(\Gamma)$. The following theorem is a result of this type, and will be proved in Section 5. It was Cai Heng Li who suggested that it might be true, and suggested a strategy for its proof.

THEOREM 1. Let $G$ be a nonabelian simple group, and suppose that $\Gamma=$ $\operatorname{Cay}(G, S)$ is a connected undirected graph of valency 3 . If $\Gamma$ is normal edge-transitive as an undirected graph, then $\Gamma$ is normal, that is, $\operatorname{Aut}(\Gamma)=G \cdot \operatorname{Aut}(G ; S)$.

\section{Decomposing Cayley graphs and undirected graphs}

Let $\Gamma=\operatorname{Cay}(G, S)$ be a Cayley graph for a finite group $G$, let $E(S)$ denote the set of edges of $\Gamma$, and let $N$ be a subgroup of $\operatorname{Aut}(\Gamma)$ which contains $G$. Since $G$ acts regularly on vertices, we have $N=G N_{0}$ and $N_{0} \cap G=\left\{1_{G}\right\}$, where $N_{0}$ is the stabiliser in $N$ of the vertex $1_{G} \in G$. For $g \in G$, let $\Gamma(g)$ denote the set of vertices $h$ such that $(g, h) \in E(S)$, that is, the set of out-neighbours of $g$ in $\Gamma$. Then $\Gamma(g)=\left\{h: h g^{-1} \in S\right\}=S g$, so in particular, $\Gamma\left(1_{G}\right)=S$.

Suppose that, in its action on $E(S)$, the group $N$ has orbits $E_{1}, \ldots, E_{r}$. Then the sets $S_{i}:=\left\{h \mid\left(1_{G}, h\right) \in E_{i}\right\}$, for $1 \leqslant i \leqslant r$, are the orbits of $N_{0}$ in $\Gamma\left(1_{G}\right)$. Moreover, for $1 \leqslant i \leqslant r$, the Cayley graph Cay $\left(G, S_{i}\right)$ has edge set $E_{i}$ and admits $N$ as an edgetransitive subgroup of automorphisms. Thus we may partition the edge set of $\operatorname{Cay}(G, S)$ in such a way that each part of the partition is the edge set of a Cayley graph for $G$ which admits the group $N$ acting edge-transitively. 
Moreover if $\operatorname{Cay}(G, S)$ is undirected, that is, if $S$ is symmetric, then we may also partition the edge set of $\operatorname{Cay}(G, S)$ in such a way that each part of the partition is the edge set of an undirected Cayley graph for $G$ which admits the group $N$ acting transitively on unordered edges. We do this as follows. There is a natural pairing on the $E_{i}$ and on the $S_{i}$. The set of edges paired with $E_{i}$ is defined as $E_{i}^{\prime}:=\left\{(h, g) \mid(g, h) \in E_{i}\right\}$, and the subset of $S$ paired with $S_{i}$ is defined as $S_{i}^{\prime}:=\left\{h \mid\left(1_{G}, h\right) \in E_{i}^{\prime}\right\}$. It is straightforward to show that $E_{i}^{\prime}$ is an $N$-orbit in $E(S)$ so that $E_{i}^{\prime}=E_{i^{\prime}}$ for some $i^{\prime}$ with $1 \leqslant i^{\prime} \leqslant r$, and hence that $S_{i}^{\prime}=S_{i^{\prime}}$ is an $N_{0}$-orbit in $S$. Moreover we have the following.

LEMma 1. Suppose that $\operatorname{Cay}(G, S)$ is undirected. Then, with the notation above, for each $i=1, \ldots, r$, the subset $S_{i^{\prime}}$ paired with $S_{i}$ is equal to $S_{i}^{-1}$, and hence Cay $(G$, $\left.S_{i} \cup S_{i^{\prime}}\right)$ is undirected and $N$ is transitive on its unordered edges.

Proof: Note $g \in S_{i}^{\prime}$ if and only if $\left(1_{G}, g\right) \in E_{i}^{\prime}$ which, by definition, holds if and only if $\left(g, 1_{G}\right) \in E_{i}$. Applying $g^{-1} \in G$ to this pair, we see that the latter inclusion holds if and only if $\left(1_{G}, g^{-1}\right) \in E_{i}$, that is, if and only if $g^{-1} \in S_{i}$. Hence $S_{i}^{\prime}=S_{i}^{-1}$ (which is in particular an $N_{0}$-orbit in $S$ ), so $T:=S_{i} \cup S_{i}^{\prime}$ is symmetric and Cay $(G, T)$ is undirected. Moreover if $(g, h)$ and $\left(g^{\prime}, h^{\prime}\right)$ are edges of $\operatorname{Cay}(G, T)$ then one of $\left(g^{\prime}, h^{\prime}\right)$ and $\left(h^{\prime}, g^{\prime}\right)$ lies in the $N$-orbit on edges which contains $(g, h)$, and hence some element of $N$ maps the unordered edge $\left\{\left(g^{\prime}, h^{\prime}\right),\left(h^{\prime}, g^{\prime}\right)\right\}$ to the unordered edge $\{(g, h),(h, g)\}$.

Clearly the paired orbit of $E_{i}^{\prime}$ and the paired subset of $S_{i}^{\prime}$ are equal to $E_{i}$ and $S_{i}$ respectively. It is possible for $E_{i}^{\prime}$ to be equal to $E_{i}$ and in this case $S_{i}$ is symmetric, $\operatorname{Cay}\left(G, S_{i}\right)$ is undirected, and $N$ is transitive on its edges. We have therefore proved the following result.

Theorem 2. Let $\Gamma=\operatorname{Cay}(G, S)$ be a Cayley graph for a finite group $G$, and let $N$ satisfy $G \leqslant N \leqslant \operatorname{Aut}(\Gamma)$. Then we may partition the edge set of $\Gamma$ into edge sets for Cayley graphs $\Gamma_{1}, \ldots, \Gamma_{r}$ for $G$ such that $N$ is transitive on the edges of each of the $\Gamma_{i}$. Moreover, if $\Gamma$ is undirected then we may alternatively choose each of the $\Gamma_{i}$ to be undirected with $N$ transitive on its unordered edges.

Note that Theorem 2 holds for any subgroup $N$ containing $G$. If, in Theorem $2, N$ normalises $G$, then the graphs $\Gamma_{i}$ are all normal edge-transitive Cayley graphs, or normal edge-transitive as undirected graphs if $\Gamma$ is undirected. In the next section we shall examine certain quotient graphs of an edge-transitive Cayley graph $\Gamma$ for a group $G$, and we shall see that in order to guarantee that the quotient graphs are also edge-transitive Cayley graphs it is appropriate to choose $N$ to normalise $G$.

In the remainder of this section we give a preliminary discussion of normal edgetransitive Cayley graphs $\Gamma=\operatorname{Cay}(G, S)$. First we make some comments about equation (1). The group $N_{\mathrm{Sym}(G)}(G)$ is called the holomorph of $G$, and is the semidirect product $G \cdot \operatorname{Aut}(G)$, where elements of $\operatorname{Aut}(G)$ have the natural conjugation action on the normal subgroup $G$ (that is, $\sigma^{-1} g \sigma=g^{\sigma}$ for $g \in G, \sigma \in \operatorname{Aut}(G)$ ), and also act naturally as 
permutations of $G$ (that is, $\sigma: g \mapsto g^{\sigma}$ ). The subgroup Aut $(G)$ is the stabiliser of $1_{G}$ in $N_{\mathrm{Sym}(G)}(G)$. Thus, when $G \leqslant N \leqslant N_{\text {Aut }(\Gamma)}(G)$, we have $N=G N_{0}$, where $N_{0} \leqslant$ Aut $(G)$. In particular, it is not difficult to prove that $N_{\mathrm{Aut}(\Gamma)}(G)=G \cdot \operatorname{Aut}(G ; S)$ as in (1). It is possible to characterise normal edge-transitivity in terms of the action of $\operatorname{Aut}(G ; S)$.

Proposition 1. Let $\Gamma=\operatorname{Cay}(G, S)$ be a Cayley graph for a finite group $G$ with $S \neq \emptyset$, and let $N=G \cdot N_{0}$ where $N_{0} \leqslant \operatorname{Aut}(G ; S)$. Then

(a) $N$ is transitive on edges if and only if $S$ is an $N_{0}$-orbit;

(b) if $\Gamma$ is undirected, then $N$ is transitive on unordered edges if and only if either $S$ is an $N_{0}$-orbit, or $S$ is the disjoint union of sets $T$ and $T^{-1}$ where both $T$ and $T^{-1}$ are $N_{0}$-orbits;

(c) $\Gamma$ is normal edge-transitive if and only if $\operatorname{Aut}(G ; S)$ is transitive on $S$, and if $\Gamma$ is undirected, then $\Gamma$ is normal edge-transitive as an undirected graph if and only if $\operatorname{Aut}(G ; S)$ is either transitive on $S$, or has two orbits in $S$ which are inverses of each other.

Proof: We give a proof of part (b). The proof of part (a) is similar and easier, and part (c) follows immediately from parts (a) and (b). As discussed above, $N_{0}$ acts on vertices by conjugation, and fixes setwise the set $S$ of out-neighbours of $1_{G}$. Suppose that $N$ is transitive on unordered edges, and let $s, s^{\prime} \in S$. Then, $\left(1_{G}, s\right)$ and $\left(1_{G}, s^{\prime}\right)$ are edges of $\Gamma$ so there is an element $n=g n_{0} \in N$ which maps the unordered edge containing $\left(1_{G}, s\right)$ to the unordered edge containing $\left(1_{G}, s\right)$, with $g \in G, n_{0} \in N_{0}$. If $g=1_{G}$, then $s^{n_{0}}=s^{\prime}$. On the other hand if $g \neq 1_{G}$ then $g$ must map $s$ to $1_{G}$, so $g=s^{-1}$, and $n_{0}$ maps $s^{-1}$ to $s^{\prime}$. Thus $s^{\prime}$ lies in the $N_{0}$-orbit containing $s$ or $s^{-1}$.

Conversely, suppose that $N_{0}$ is either transitive on $S$, or has two orbits in $S$ which are inverses of each other. For any two unordered edges $e, e^{\prime}$, we may apply elements of $G$ to $e, e^{\prime}$ so that the images are unordered edges which contain $\left(1_{G}, s\right)$ and $\left(1_{G}, s^{\prime}\right)$, say, where $s, s^{\prime} \in S$. By our assumption on $N_{0}$, some element of $N_{0}$ will map $\left(1_{G}, s\right)$ to $\left(1_{G}, s^{\prime}\right)$ or $\left(1_{G},\left(s^{\prime}\right)^{-1}\right)$, and in the latter case further application of $s^{\prime} \in G$ will map this ordered pair to $\left(s^{\prime}, 1_{G}\right)$. Thus $e$ and $e^{\prime}$ lie in the same $N$-orbit on unordered edges.

Finally we make a few remarks concerning the connectivity of graphs occurring in the decomposition process described above. Similar remarks apply in the undirected case.

REMARK 1. If Cay $(G, S)$ is a connected Cayley graph, then in general the subgraphs $\operatorname{Cay}\left(G, S_{i}\right)$ defined above need not be connected. However it is straightforward to check that each connected component of $\operatorname{Cay}\left(G, S_{i}\right)$ will be isomorphic to the connected Cayley graph Cay $\left(\left\langle S_{i}\right\rangle, S_{i}\right)$, and that the latter graph admits a (possibly unfaithful) edgetransitive action of the subgroup $N_{N}\left(\left\langle S_{i}\right\rangle\right)$ of $N$. This means that in some circumstances we may assume that the Cayley graphs are connected as well as normal edge-transitive. However some care may be needed in extracting information about general Cayley graphs from similar information about connected normal edge-transitive Cayley graphs. 


\section{Quotients of CAyley graphs and digraphS}

For a graph $\Gamma$ with vertex set $V$, and a partition $\mathcal{P}$ of $V$, the quotient graph $\Gamma_{\mathcal{P}}$ of $\Gamma$ with respect to $\mathcal{P}$ is defined to have vertex set $\mathcal{P}$, with $\left(p, p^{\prime}\right)$ an edge of $\Gamma_{\mathcal{P}}$ if and only if there are vertices $x \in p$ and $x^{\prime} \in p^{\prime}$ such that $\left(x, x^{\prime}\right)$ is an edge of $\Gamma$. If $\Gamma$ is undirected then clearly $\Gamma_{\mathcal{P}}$ will be undirected also.

Now consider the special case where $\Gamma$ is a Cayley graph $\operatorname{Cay}(G, S)$. Here $\mathcal{P}$ is a partition of the group $G$. Moreover the regular subgroup $G$ of $\operatorname{Aut}(\Gamma)$ is our evidence that $\Gamma$ is a Cayley graph. The quotient graphs which will be of particular interest to us are those which we know to be Cayley graphs because we know regular subgroups of automorphisms of them which are related to the group $G$. The next result shows that $\Gamma_{\mathcal{P}}$ will be the Cayley graph of a group associated with $G$ precisely when $\mathcal{P}$ is the set of cosets of a normal subgroup of $G$. For $g \in G$ let $\mathcal{P}(g)$ denote the part of $\mathcal{P}$ containing the element $g$.

Theorem 3. Suppose that $\mathcal{P}$ is a partition of the vertex set $G$ of the Cayley graph $\Gamma=\operatorname{Cay}(G, S)$.

(a) Then $G$ induces a group of automorphisms of $\Gamma_{\mathcal{P}}$ (such that, for $x, g \in G$, $x: \mathcal{P}(g) \mapsto \mathcal{P}(g x))$ if and only if $\mathcal{P}$ is the set $[G: H]$ of right cosets of a subgroup $H$ of $G$.

(b) If $\mathcal{P}=[G: H]$ for some subgroup $H$, then the automorphism group induced by $G$ on $\Gamma_{\mathcal{P}}$ is regular if and only if $H$ is normal, and in this case $\Gamma_{\mathcal{P}}=$ $\operatorname{Cay}(G / H, S H / H)$.

(c) If $\mathcal{P}=[G: H]$ for some normal subgroup $H$, and if $N=G N_{0}$ where $N_{0} \leqslant \operatorname{Aut}(G ; S)$, then $N$ induces a group of automorphisms of $\Gamma_{\mathcal{P}}$ if and only if $H$ is $N_{0}$-invariant. Moreover, in this case, if $N$ is edge-transitive on $\Gamma$ then $N$ is also edge-transitive on $\Gamma_{\mathcal{P}}$; and if $\Gamma$ is undirected and $N$ is transitive on the unordered edges of $\Gamma$, then $N$ is also transitive on the unordered edges of $\Gamma_{\mathcal{p}}$.

Proof: Suppose that, for $x, g \in G, x$ maps $\mathcal{P}(g)$ to $\mathcal{P}(g x)$. Set $H:=\mathcal{P}\left(1_{G}\right)$. Then $x$ maps $H$ to $H x$ and so $\mathcal{P}(x)=H x$, for all $x \in G$. In particular, for $h, h^{\prime} \in H$, $\mathcal{P}(h)=\mathcal{P}\left(h^{\prime}\right)=\mathcal{P}\left(1_{G}\right)=H$. Since $h^{-1}$ maps $H=\mathcal{P}(h)$ to $\mathcal{P}\left(h h^{-1}\right)=\mathcal{P}\left(1_{G}\right)=H$, it follows that $H h^{-1}=H$, so $h^{\prime} h^{-1} \in H$. Thus $H$ is a subgroup of $G$ and $\mathcal{P}$ is the set of right cosets of $H$. Conversely if $\mathcal{P}$ is the set of right cosets of a subgroup $H$, so that $\mathcal{P}(g)=H g$ for $g \in G$, then $G$ induces an action on $\mathcal{P}$ by right multiplication and it is straightforward to check that $G$ preserves adjacency in $\Gamma_{\mathcal{P}}$ so that $G$ induces a group of automorphisms of $\Gamma_{\mathcal{P}}$.

Now let $H$ be a subgroup of $G$ and $\mathcal{P}=[G: H]$. The group induced by $G$ on $\Gamma_{\mathcal{P}}$ is regular if and only if the setwise stabiliser of $\mathcal{P}\left(1_{G}\right)$ in $G$, namely $H$, fixes all parts of $\mathcal{P}$ setwise, that is, $H g h=H g$ for all $g \in G$ and $h \in H$. This is true if and only if $H$ is normal 
in $G$, and in this case $\Gamma_{\mathcal{P}}$ is a Cayley graph for $G / H$. In this case, $\left(H g, H g^{\prime}\right)$ is an edge of $\Gamma_{\mathcal{P}}$ if and only if, for some $h, h^{\prime} \in H,\left(h g, h^{\prime} g^{\prime}\right)$ is an edge of $\Gamma$, that is $h^{\prime} g^{\prime}(h g)^{-1} \in S$, which is equivalent to $g^{\prime} g^{-1} \in\left(h^{\prime}\right)^{-1} S h \subseteq S H$. Thus $\Gamma_{\mathcal{P}}=\operatorname{Cay}(G / H, S H / H)$.

Suppose now that $H$ is normal in $G$, so that $\Gamma_{\mathcal{P}}=\operatorname{Cay}(G / H, S H / H)$, and let $N=G N_{0}$ with $N_{0} \leqslant \operatorname{Aut}(G ; S)$. Then $N$ acts as a group of automorphisms of $\Gamma_{\mathcal{P}}$ if and only if $N_{0}$ does. If $N_{0}$ acts as a group of automorphisms of $\Gamma_{\mathcal{P}}$, then $N_{0}$ leaves invariant $\mathcal{P}\left(l_{G}\right)=H$. Conversely, suppose that $H$ is $N_{0}$-invariant, and that $(H x, H y)$ is an edge of $\Gamma_{\mathcal{P}}$. Then $(H y)(H x)^{-1}=H y x^{-1} \in H S / H$, say $y x^{-1}=h s$ where $h \in H, s \in S$, and since both $H$ and $S$ are $N_{0}$-invariant, it follows that, for $n \in N_{0},\left(H y x^{-1}\right)^{n}=H h^{n} s^{n}=$ $H s^{n} \in H S / H$, and hence that $\left(H x^{n}, H y^{n}\right)$ is an edge of $\Gamma_{P}$. Thus we have shown that $N$ acts as a group of automorphisms of $\Gamma_{\mathcal{P}}$ if and only if $H$ is $N_{0}$-invariant. Finally, verifying the assertions about transitivity of $N$ on edges and unordered edges of $\Gamma_{\mathcal{p}}$ is straightforward.

We can now prove the assertions made in the introduction about the family $\mathcal{C}(G, S)$ of quotient graphs of a normal edge-transitive Cayley graph Cay $(G, S)$ modulo $\operatorname{Aut}(G ; S)$ invariant subgroups of $G$. A subgroup $H$ of a group $G$ is said to be characteristic if $H$ is invariant under the full automorphism group $\operatorname{Aut}(G)$. In particular characteristic subgroups are normal, and the identity subgroup is a proper characteristic subgroup of a nontrivial group $G$. A nontrivial group $G$ is said to be characteristically simple if its only characteristic subgroups are the identity subgroup and the group $G$. It is well known, see for example [5, 2.1.4], that a finite group is characteristically simple if and only if it is a direct product of isomorphic simple groups. Suppose that $N=G N_{0}$ with $N_{0} \leqslant \operatorname{Aut}(G ; S)$. Then each characteristic subgroup of $G$ is $N_{0}$-invariant. Consequently if $H$ is a maximal $N_{0}$-invariant subgroup of $G$, then $G / H$ is characteristically simple since if $L / H$ is a characteristic subgroup of $G / H$, where $H \leqslant L \leqslant G$, then $L$ is a characteristic subgroup of $G$. Also, if $H$ is a maximal $N_{0}$-invariant subgroup of $G$, then $G / H$ is a minimal normal subgroup of $(G / H) \cdot \widehat{N_{0}}$ where $\widehat{N_{0}}$ is the subgroup of Aut $(G / H)$ induced by $N_{0}$. For $N_{0}$-invariant subgroups $L, H$ such that $L \leqslant H<G$, there is a natural epimorphism $G / L \rightarrow G / H$ which maps $S L / L$ to $S H / H$, and therefore induces a graph homomorphism from $\operatorname{Cay}(G / L, S L / L)$ onto $\operatorname{Cay}(G / H, S H / H)$. We shall say that an element $\operatorname{Cay}(G / H, S H / H) \in \mathcal{C}(G, S)$ is $N$-minimal if $H$ is a maximal $N_{0}$ invariant subgroup of $G$. Thus $\operatorname{Cay}(G / H, S H / H)$ is $N$-minimal if and only if the only graph homomorphism $\mathrm{Cay}(G / L, S L / L) \rightarrow \operatorname{Cay}(G / H, S H / H)$ as above is the identity homomorphism. Thus we have proved the following.

THEOREM 4. Let $\Gamma=\operatorname{Cay}(G, S)$, for a nontrivial finite group $G$, and let $N=$ $G N_{0}$, where $N_{0} \leqslant \operatorname{Aut}(G ; S)$, be such that either $N$ is edge-transitive on $\Gamma$, or $\Gamma$ is undirected and $N$ is transitive on unordered edges. Then the family $\mathcal{C}(G, S)$ is nonempty. Moreover,

(a) if $\operatorname{Cay}(G / H, S H / H) \in \mathcal{C}(G, S)$ is $N$-minimal, then $G / H$ is characteristi- 
cally simple and is a minimal normal subgroup of $(G / H) \cdot \widehat{N_{0}}$, where $\widehat{N_{0}}$ is the subgroup of $\operatorname{Aut}(G / H ; S H / H)$ induced by $N_{0} ;$ and

(b) for every $\Gamma_{\mathcal{P}}=\operatorname{Cay}(G / L, S L / L) \in \mathcal{C}(G, S)$ there is at least one graph homomorphism from $\Gamma_{\mathcal{P}}$ onto an $N$-minimal element of $\mathcal{C}(G, S)$.

\section{Constructing NORMAL EdGe-TRANSITIVE CAYLEY GRAPHS FROM QUOTIENTS}

In this section we give a general answer to Question 1. Suppose that the following hypothesis holds.

HYPothesis 1. Let $\Sigma=\operatorname{Cay}(\widehat{G}, \widehat{S})$ be a Cayley graph for a finite nontrivial group $\widehat{G}$, and let $\widehat{N}=\widehat{G} \widehat{N_{0}}$, where $\widehat{N_{0}} \leqslant \operatorname{Aut}(\widehat{G}, \widehat{S})$, be such that either (i) $\widehat{N}$ is edge-transitive on $\Sigma$, or (ii) $\Sigma$ is undirected and $\widehat{N}$ is transitive on the unordered edges of $\Sigma$. Suppose further that $G$ is a group with normal subgroup $H$ such that $G / H=\widehat{G}$.

We wish to know if the following is true.

CONCLusion 1. There is a subgroup $N=G N_{0}$ such that $N_{0} \leqslant N_{\mathrm{Aut}(G)}(H)$, and there is a Cayley graph $\operatorname{Cay}(G, S)$ for $G$ such that $S H / H=\widehat{S}$, and either $N$ is edge-transitive on Cay $(G, S)$, or Cay $(G, S)$ is undirected and $N$ is transitive on the unordered edges of Cay $(G, S)$, according as (i) or (ii) of Hypothesis 1 holds.

For Conclusion 1 to hold, there is a necessary condition on $\widehat{N}$ which can be described as follows. Observe that the natural action of $\operatorname{Aut}(G)$ on $G$ induces a homomorphism $\phi: N_{\text {fut }(G)}(H) \rightarrow \operatorname{Aut}(\widehat{G})$. Set $\widehat{M}_{0}:=\phi\left(N_{\text {Aut }(G)}(H)\right) \cap \operatorname{Aut}(\Sigma)$.

Lemma 2. Suppose that Hypothesis 1 holds. A necessary condition for Conclusion 1 to hold is that $\widehat{G} \widehat{M}_{0}$ is transitive on edges or unordered edges of $\Sigma$, according as (i) or (ii) of Hypothesis 1 holds.

Proof: Suppose that such a subgroup $N$ and graph $\operatorname{Cay}(G, S)$ exist. Then, by Theorem 3 (c), $N$ is transitive on the edges or unordered edges of $\Sigma$, according as (i) or (ii) holds. The group induced by $N$ on $\Sigma$ is $\widehat{G} \phi\left(N_{0}\right)$. Since $\phi\left(N_{0}\right) \subseteq \widehat{M}_{0}$, it follows that $\widehat{G} \widehat{M}_{0}$ is transitive on the edges or unordered edges of $\Sigma$ respectively.

We show below that the necessary condition of Lemma 2 is also sufficient, and we describe the class of all normal edge-transitive Cayley graphs for $G$ which correspond to the given graph $\Sigma$. Recall that, by Proposition 1, if $\widehat{G} \widehat{M}_{0}$ is edge-transitive on $\Sigma$, then $\widehat{M}_{0}$ is transitive on $\widehat{S}$, and if $\widehat{G} \widehat{M}_{0}$ is transitive on the unordered edges of $\Sigma$, then either $\widehat{M}_{0}$ is transitive on $\widehat{S}$, or $\widehat{S}=\widehat{T} \cup \widehat{T}^{-1}$ with $\widehat{T}$ an $\widehat{M}_{0}$-orbit. For $s \in G$ and $X \leqslant \operatorname{Aut}(G)$ we denote the $X$-orbit containing $s$ by $s^{X}$, that is, $s^{X}=\left\{s^{x} \mid x \in X\right\}$.

THEOREM 5. Suppose that Hypothesis 1 holds. Then Conclusion 1 holds if and only if $\widehat{G} \widehat{M}_{0}$ is transitive on edges or unordered edges of $\Sigma$, according as (i) or (ii) of Hypothesis 1 holds. Suppose then that this condition on $\widehat{G} \widehat{M}_{0}$ is satisfied, and let $M_{0}:=\phi^{-1}\left(\widehat{M}_{0}\right)$ be the full pre-image of $\widehat{M}_{0}$. 
(a) Let $N_{0}$ be a subgroup of $M_{0}$ such that $\widehat{G} \phi\left(N_{0}\right)$ is transitive on edges or unordered edges of $\Sigma$, according as (i) or (ii) of Hypothesis 1 holds. Let $s \in G$ be such that $H s \in \widehat{S}$. Let $S=s^{N_{0}}$ if (i) holds, and $S=s^{N_{0}} \cup\left(s^{N_{0}}\right)^{-1}$ if (ii) holds. Then $S H / H=\widehat{S}$, and the conditions of Conclusion 1 are satisfied for $N:=G N_{0}$ and $\operatorname{Cay}(G, S)$.

(b) All subgroups $N$ and graphs $\operatorname{Cay}(G, S)$ for which the conditions of Conclusion 1 hold can be constructed as in part (a).

Proof: Suppose that $\widehat{G} \widehat{M}_{0}$ is transitive on edges or unordered edges of $\Sigma$, according as (i) or (ii) of Hypothesis 1 holds. Let $N_{0}$ and $S$ be as in part (a). By the definition of $S$ and Proposition 1, it follows that $S H / H=\widehat{S}$, that $S$ is symmetric if (ii) holds, and that the conditions of Conclusion 1 hold for $N$ and Cay $(G, S)$. This proves part (a), and also proves that the condition on $\widehat{G} \widehat{M}_{0}$ in Lemma 2 is sufficient for Conclusion 1 to hold.

Conversely, suppose that Conclusion 1 holds for $N=G N_{0}$ and $\operatorname{Cay}(G, S)$. Then $S H / H=\widehat{S}$. By Theorem 3 (c), $N$ induces a group of automorphisms of $\Sigma$, and hence in particular, $N_{0} \leqslant M_{0}$. Also, by Theorem $3(\mathrm{c}), \widehat{G} \phi\left(N_{0}\right)$ has the transitivity properties required in part (a). By Proposition $1, S=s^{N_{0}}$ if (i) holds, and $S=s^{N_{0}} \cup\left(s^{N_{0}}\right)^{-1}$ if (ii) holds. Thus $N$ and $\operatorname{Cay}(G, S)$ can be constructed as in part (a).

Houlis [6] used essentially the strategy described in this section to classify all $N$ and $\operatorname{Cay}(G, S)$ arising in Conclusion 1 in the case where $\widehat{G}=\mathrm{Z}_{p}$ with $p$ a prime, $\widehat{S} \neq \emptyset$, and $G=\mathrm{Z}_{p} \times \mathrm{Z}_{p}, \mathbf{Z}_{p^{2}}$, or $\mathbf{Z}_{p} \times \mathbf{Z}_{q}$ for a prime $q \neq p$. In this case, by Example $2, \Sigma=\Gamma(p ; a)$ for some divisor $a$ of $p-1$, with $p$ or $a$ even if $\Sigma$ is undirected. (Houlis [6] considered only lindirected Cayley graphs.)

Since $\Sigma$ is connected, the disconnected examples $\operatorname{Cay}(G, S)$ are isomorphic to $|G| / p$ copies of $\Sigma$. So we may assume that $\operatorname{Cay}(G, S)$ is connected. Since all the groups $G$ considered are Abelian, the map $\sigma$ which inverts every element of $G$ lies in $\operatorname{Aut}(G)$. If $\Sigma$ is undirected, and we are seeking symmetric subsets $S$, we may assume that $\sigma \in \operatorname{Aut}(G ; S)$. Hence in all cases $\widehat{M}_{0}$ is transitive on $\widehat{S}$, and so $\widehat{M}_{0} \cong \mathrm{Z}_{a}$.

In the cases $G=\mathrm{Z}_{p^{2}}$ and $G=\mathbf{Z}_{p q}$, there is a unique subgroup $H$ such that $G / H \cong \widehat{G}$, and hence $H$ is invariant under $\operatorname{Aut}(G)$. Hence the classification gives all normal edgetransitive Cayley graphs for these groups. In the former case, $M_{0} \cong \mathbf{Z}_{p a}$, and $N_{0}$ is any subgroup of $M_{0}$ for which $\phi\left(N_{0}\right)=\widehat{M}_{0}$, that is, $N_{0} \cong M_{0}$ or $\mathbf{Z}_{a}$. For each of these possibilities Houlis showed that there is up to isomorphism only one possibility for $S$ (as we may always assume that $1 \in S$ ). Thus we get two possible graphs $\operatorname{Cay}(G, S)$ for each $\Sigma=\Gamma(p ; a)$. This was proved by Houlis in $[6$, Chapter 7], and is the easiest of the three cases. If $G=\mathbf{Z}_{p q}$ with $q \neq p$, then $\operatorname{Aut}(G) \cong \mathbf{Z}_{p-1} \times \mathbf{Z}_{q-1}$, and $M_{0} \cong \mathbf{Z}_{a} \times \mathbf{Z}_{q-1}$. The group $N_{0}$ can be any subgroup of $M_{0}$ which projects onto $\mathbf{Z}_{a}$ in the first factor. In this case the group $G$ also has a unique quotient group isomorphic to $\mathbf{Z}_{q}$, and the normal edgetransitive graphs $\operatorname{Cay}(G, S)$ will have also a normal edge-transitive quotient isomorphic to $\Gamma(q ; b)$ for some divisor $b$ of $q-1$, with $q$ or $b$ even if the graphs are undirected. Thus 
it is helpful to use $a$ and $b$ as parameters of the graphs $\operatorname{Cay}(G, S)$. For a given $a, b$, the subgroup $N_{0}$ is a subdirect product of $\mathbf{Z}_{a} \times \mathbf{Z}_{b}$, and each such subdirect product corresponds to a normal edge-transitive graph $\operatorname{Cay}(G, S)$. The subdirect products of $\mathbf{Z}_{a} \times \mathbf{Z}_{b}$, and hence the connected normal edge-transitive Cayley graphs for $\mathbf{Z}_{p q}$ were parametrised in [6, Chapter 8].

If $G=Z_{p} \times Z_{p}$, then as $\operatorname{Aut}(G) \cong G L(2, p)$ is transitive on the $p+1$ subgroups of $G$ of order $p$, we may assume that $H=\langle(1,0)\rangle$, and then we have

$$
M_{0}=\left\{\left(\begin{array}{ll}
b & 0 \\
c & d
\end{array}\right) \mid b d \neq 0, d^{a} \equiv 1 \quad(\bmod p)\right\}
$$

and

$$
\phi:\left(\begin{array}{cc}
b & 0 \\
c & d
\end{array}\right) \mapsto d
$$

Houlis [6, Chapter 6] showed that either $\operatorname{Cay}(G, S)$ is the lexicographic product $\Gamma(p ; a)\left[\overline{K_{p}}\right]$, or $N_{0}$ is a subdirect product of $\mathbf{Z}_{p-1} \times \mathbf{Z}_{a}$. In the latter case, $G$ has a second $N_{0}$-invariant subgroup of order $p$, and hence a second quotient, say $\Gamma(p ; b)$. For fixed $a, b$, the group $N_{0}$ is a subdirect product of $\mathbf{Z}_{b} \times \mathbf{Z}_{a}$, and the possibilities for $N_{0}$ and $\operatorname{Cay}(G, S)$ were parametrised, and isomorphisms identified by Houlis in a similar way to the previous case. For $G=\mathbf{Z}_{p} \times \mathbf{Z}_{p}$, this classification gives all normal edge-transitive Cayley graphs $\operatorname{Cay}(G, S)$ such that $\operatorname{Aut}(G ; S)$ acts reducibly on $G$.

\section{Proof OF Theorem 1}

Let $G$ be a finite nonabelian simple group, and suppose that $\Gamma=\operatorname{Cay}(G, S)$ is a connected undirected graph of valency 3 , which is normal edge-transitive as an undirected graph. Then $S$ is symmetric of size 3 , and $S$ generates $G$. Let $N=G \cdot N_{0}$, where $N_{0}=\operatorname{Aut}(G ; S)$. It follows from Proposition 1 that $S$ is an $N_{0}$-orbit, so in particular $\left|N_{0}\right|$ is divisible by 3. Also, the action of $N_{0}$ on $S$ is faithful, and hence $\left|N_{0}\right|=3$ or 6 .

Set $A:=\operatorname{Aut}(\Gamma)$, and suppose that $A \neq N$ (that is, that $\Gamma$ is not normal). By (1), $N=N_{A}(G)$. Since $G$ is transitive on vertices, we have $A=G A_{0}$, where $A_{0}$ is the stabiliser in $A$ of the vertex $1_{G}$. Thus $N_{0}<A_{0}$. Since $N$, and hence also $A$, are transitive on edges (which are ordered pairs of vertices), it follows from a result of Tutte, see [2, Theorem 18.6], that $\left|A_{0}\right|=3 \cdot 2^{t}$, where $1 \leqslant t \leqslant 4$. In particular $A_{0}$ is soluble, and $n:=|A: N|$ divides 16 .

Let $M=\bigcap_{a \in A} N^{a}$, the core of $N$ in $A$. Then $M$ is the kernel of the action of $A$ by right multiplication on the set $\Omega:=[A: N]$ of right cosets of $N$ in $A$. Thus $A / M$ is isomorphic to a subgroup $\widehat{A}$ of the symmetric group $S_{n}$. Now $G$ is a characteristic subgroup of $N$. If $G$ were contained in $M$, then it would be characteristic in $M$ and hence normal in $A$, 
and this contradicts our assumption. Hence $G \cap M=1$, so $M \times G \leqslant N$, whence $|M|$ divides 6 and $n|M|_{2} \leqslant 16$. The stabiliser $\widehat{N}=N / M$ in $\widehat{A}$ has a subgroup isomorphic to $G$, and hence $\widehat{A}$ is insoluble, so $n=8$ or 16 . Thus $\left|A_{0}\right|=24$ or $48, A_{0} \cong S_{4}$ or $S_{4} \times Z_{2}$, and $A$ is transitive on the 4 -arcs or 5 -arcs of $\Gamma$ respectively, (see [2,18C on p.26]). In particular some element of $A_{0}$ inverts the subgroup $X_{0}$ of $N_{0}$ of order 3.

Suppose first that 3 divides $|M|$. Then $M$ has a characteristic subgroup $X$ of order 3 , and $X_{0}$ is contained in $X \times G$ and projects nontrivially onto each factor. Since some element of $A_{0}$ inverts $X_{0}$, it follows that $A$ inverts $X$. Hence the centraliser $A_{1}:=C_{A}(X)$ has index 2 in $A$. Thus we have $N_{1}:=N \cap A_{1}=X \times G$ and $M \cap A_{1}=X$. Now $\left|A: N_{1}\right|=|A: G| / 3$ divides 16 , and hence $\left|A_{1}: N_{1}\right|$, which is the length of the orbits of $\widehat{A_{1}}=A_{1} M / M$ in $\Omega$, divides 8 . Since $\widehat{A_{1}}$ is insoluble we have $\left|A_{1}: N_{1}\right|=8$. Since the group induced by $\widehat{A_{1}}$ on each of its orbits in $\Omega$ is insoluble, and since $\widehat{A_{1}}$ has a unique insoluble composition factor (since $\left|\widehat{A_{1}}: \widehat{G}\right|$ divides 16 ), it follows that $\widehat{G}$ acts nontrivially on each $\widehat{A_{1}}$-orbit. A transitive permutation group of degree 8 with insoluble point stabiliser must be primitive and, for example by [11, Table 1], must be one of $S_{8}, A_{8}$, or $\operatorname{AGL}(3,2)$. Since the point stabiliser $\widehat{N_{1}}=\widehat{G}$ is simple, $\widehat{A_{1}}=A_{8}$ or $\operatorname{AGL}(3,2)$, $G=A_{7}$ or $\mathrm{GL}(3,2)$, and $A=\left(\mathrm{Z}_{3} \times A_{8}\right) \cdot 2$ or $\left(\mathrm{Z}_{3} \times \mathrm{AGL}(3,2)\right) \cdot 2$ respectively. In either case $\left|A_{0}\right|=48$, so $A_{0}=S_{4} \times Z_{2}$, and $A$ is transitive on the 5 -arcs of $\Gamma$. In the former case, $A$ has a normal subgroup $Y=A_{8}$ which is vertex-transitive (because $G<Y$ ), and the stabiliser of $1_{G}$ in $Y$ is $Y_{0}=Y \cap A_{0}$ of order $|Y: G|=8$. The group induced by $Y_{0}$ on $\Gamma\left(1_{G}\right)$ is therefore a normal 2-subgroup of the group induced by $A_{0}$ on $\Gamma\left(1_{G}\right)$. Since the latter group is $S_{3}$, it follows that $Y_{0}$ acts trivially on $\Gamma\left(1_{G}\right)$. This however contradicts the connectivity of $\Gamma$. In the latter case, $A$ has a normal subgroup $Y=\mathrm{Z}_{2}^{3}$ which has more than 2 orbits on vertices, and so by [9, Theorem 4.1], the quotient graph $\Gamma_{Y}$ modulo the $Y$-orbits is 5-arc transitive with $|G| /|Y|=21$ vertices. There is no such graph.

Thus $|M|$ is not divisible by 3 . Since $N$ does not have a normal subgroup of order 2 , it follows that $M=1$. Thus $\widehat{A} \cong A$, and the point stabiliser $\widehat{N}$ has a simple normal subgroup $\widehat{G}$ of index 3 or 6 . As in the previous paragraph, the only transitive groups of degree 8 with insoluble point stabilisers are $S_{8}, A_{8}$, and $\operatorname{AGL}(3,2)$, and none of these have stabilisers of the required form. Hence $n=16$, and therefore $\widehat{N}=\widehat{G} \cdot 3$. Suppose that $\widehat{A}$ is imprimitive. Then $\widehat{A} \leqslant S_{u}$ wr $S_{v}$ where $u v=16$, and since $\widehat{A}$ is insoluble, $(u, v)=(2,8)$ or $(8,2)$. In the former case, the action of $\hat{A}$ on a set of 8 blocks of imprimitivity is transitive and a stabiliser is isomorphic to $\widehat{N}=\widehat{G} \cdot 3$ (since the kernel of this action is a 2-group). We have just shown that there are no such groups. In the latter case the action induced on a block of size 8 has this property, and again we have a contradiction. Hence $\hat{A}$ is primitive of degree 16. It follows from [11. Table 1] that $\widehat{A}=\mathrm{Z}_{2}^{4} \cdot \widehat{N}$ and $G=A_{5}$. Thus $\Gamma$ has 60 vertices and so the normal subgroup $Y=\mathbf{Z}_{2}^{4}$ of $A$ is intransitive with more than 2 orbits. Since $n=16, A$ acts transitively on the 5 -arcs of $\Gamma$, and it follows from [9, Theorem 4.1] that $Y$ is semiregular on vertices. This implies that 16 divides 60 , which is 
a contradiction. Thus Theorem 1 is proved.

\section{REFERENCES}

[1] Y.G. Baik, Y.Q. Feng, H.S. Sim and M.Y. Xu, 'On the normality of Cayley graphs of Abelian groups', Algebra Colloq. 5 (1998), 297-304.

[2] N. Biggs, Algebraic graph theory (Cambridge University Press, Cambridge, 1974).

[3] C.Y. Chao, 'On the classification of symmetric graphs with a prime number of vertices', Trans. Amer. Math. Soc. 158 (1971), 247-256.

[4] S.F. Du, R.J. Wang and M.Y. Xu, 'On the normality of Cayley digraphs of order twice a prime', Australas. J. Combin. 18 (1998), 227-234.

[5] D. Gorenstein, Finite groups (Harper, Evanston and London, 1968).

[6] P. Houlis, Quotients of normal-edge-transitive Cayley graphs, M.Sc. Thesis (University of Western Australia, 1998).

[7] C.H. Li, 'On isomorphisms of connected Cayley graphs, III', Bull. Austral. Math. Soc. 58 (1998), 137-145.

[8] B.D. McKay and C.E. Praeger, 'Vertex-transitive graphs which are not Cayley graphs, I', J. Austral. Math. Soc. Ser. A 56 (1994), 53-63.

[9] C.E. Praeger: 'An O'Nan-Scott Theorem for finite quasiprimitive permutation groups and an application to 2-arc transitive graphs', J. London Math. Soc. (2) 47 (1993), 227-239.

[10] C.E. Praeger, 'Finite quasiprimitive graphs', in Surveys in Combinatorics, 1997, (R.A. Bailey, Editor), London Mathematical Society Lecture Notes in Mathematics 241 (Cambridge University Press, Cambridge, 1997), pp. 65-85.

[11] C.C. Sims, 'Computational methods in the study of permutation groups', in Computational problems in abstract algebra, (J. Leech, Editor) (Pergamon Press, New York, 1970), pp. 169-184.

[12] M.Y. Xu, 'Automorphism groups and isomorphisms of Cayley graphs', Discrete Math. 182 (1998), 309-319.

Department of Mathematics and Statistics

University of Western Australia

Nedlands WA 6907

Australia 